\title{
SOME OPEN MAPPING THEOREMS FOR MARGINALS
}

\author{
BY
}

\section{LARRY Q. EIF LER}

\begin{abstract}
Let $S$ and $T$ be compact Hausdorff spaces and let $P(S), P(T)$ and $P(S \times T)$ denote the collection of probability mea sures on $S, T$ and $S \times T$, respectively. Given a probability measure $\mu$ on $S \times T$, set $\pi \mu=(a, \beta)$ where $a$ and $\beta$ are the marginals of $\mu$ on $S$ and $T$. We prove that the mapping $\pi: P(S \times T) \rightarrow P(S) \times$ $P(T)$ is norm open and weak ${ }^{*}$ open. An analogous result for $L_{1}(X \times Y, \mu \times \nu)$ where $(X, \mu)$ and $(Y, \nu)$ are probability spaces is established.
\end{abstract}

1. Introduction. Let $S$ be a compact Hausdorff space. Let $C(S)$ denote the algebra of continuous real valued functions on $S$ and let $M(S)$ denote the linear space of real valued regular Borel measures on $S$ of finite total variation. We identify $M(S)$ with the dual of $C(S)$. Let $P(S)$ denote the probability measures on $S$. In [1], Ditor and the author obtained open mapping theorems for a naturally induced mapping between spaces of probability measures on compact sets. Namely, let $S$ and $T$ be compact Hausdorff spaces and let $\phi: S \rightarrow T$ be continuous and onto. Then $\phi$ induces a mapping $\pi: P(S) \rightarrow P(T)$ defined by $\pi \mu(V)=\mu\left(\phi^{-1}(V)\right)$ for each Borel subset $V$ of $T$. The following results are established in [1].

$$
\pi: P(S) \rightarrow P(T) \text { is norm open. }
$$

One in fact obtains the following quantitative result. Let $\mu \in P(S)$ and set $a=\pi \mu$. Given $\beta \in P(T)$, there exists $\nu \in P(S)$ such that $\beta=\pi \nu$ and $\|\mu-\nu\|=\|\alpha-\beta\|$.

In this paper, we consider the open mapping properties of a naturally induced operator of interest in probability theory [3], [5]. Let $S$ and $T$ be compact Hausdorff spaces. Define $\pi: M(S \times T) \rightarrow M(S) \times M(T)$ by $\pi \mu=(\alpha, \beta)$ where $\alpha$ and $\beta$ are the marginals of $\mu$ on $S$ and $T$, i.e., $\alpha(U)=\mu(U \times T)$ and $\beta(V)=\mu(S \times V)$ for each Borel subset $U$ of $S$ and $V$ of $T$. The collection of all marginals is

Received by the editors July 12, 1973 and, in revised form, July 8, 1974. AMS (MOS) subject classifications (1970). Primary 28A35, 60B05. sures.

Key words and phrases. Marginals, open mapping theorems, probability mea- 


$$
M(S, T)=\{(\alpha, \beta) \in M(S) \times M(T): \alpha(S)=\beta(T)\} .
$$

In $\$ 2$, we establish our main results.

(1) Let $\mu \in P(S \times T)$ and set $\pi \mu=(\alpha, \beta)$. Given marginals $(\lambda, \rho)$ in $P(S) \times P(T)$, there exists $\nu \in P(S \times T)$ such that $\pi \nu=(\lambda, \rho)$ and $\|\mu-\nu\| \leq$ $\|\alpha-\lambda\|+\|\beta-\rho\|$. Hence, $\pi: P(S \times T) \rightarrow P(S) \times P(T)$ is norm open.

(2) $\pi: P(S \times T) \rightarrow P(S) \times P(T)$ is weak* open.

In $\$ 3$, we consider the analogous case of $L_{1}(X \times Y, \mu \times \nu)$ where $(X, \mu)$ and $(Y, \nu)$ are probability spaces. We also establish an open mapping result for the conditional expectation operator.

2. Probability measures on $S \times T$. In order to prove our main results (1) and (2), we first establish a quantitative version for the case when $S$ and $T$ are finite. An alternate proof of Lemma 2.1 is provided by the argument in 3.2. The type of combinatorial argument given below is needed to extend the result to finite products [6].

Lemma 2.1. Suppose $S$ and $T$ are finite sets. Let $\mu \in P(S \times T)$ and set $\pi \mu=(\alpha, \beta)$. Fix marginals $(\lambda, \rho)$ in $P(S) \times P(T)$. Then there exists $\nu \in P(S \times T)$ satisfying $\pi \nu=(\lambda, \rho)$ and $\|\mu-\nu\| \leq\|\alpha-\lambda\|+\|\beta-\rho\|$.

Proof. First consider the case where $\rho=\beta$ and where $\lambda$ and $a$ differ at exactly two points say $s_{1}$ and $s_{2}$. We may assume $\alpha\left(s_{1}\right)>\lambda\left(s_{1}\right)$ and so $\alpha\left(s_{2}\right)<\lambda\left(s_{2}\right)$. Set $\epsilon=\alpha\left(s_{1}\right)-\lambda\left(s_{1}\right)$ and $\delta=\alpha\left(s_{1}\right)$. Now define $\nu(s, t)=$ $\mu(s, t)$ if $s \neq s_{1}$ and $s \neq s_{2}$ and define $\nu\left(s_{1}, t\right)=(\delta-\epsilon) / \delta \cdot \mu\left(s_{1}, t\right)$ and $\nu\left(s_{2}, t\right)=\mu\left(s_{2}, t\right)+(\epsilon / \delta) \cdot \mu\left(s_{1}, t\right)$. Then $\pi \nu=(\lambda, \rho)$ and $\|\mu-\nu\|=2 \epsilon=$ $\|\alpha-\lambda\|$.

Now fix marginals $(\lambda, \rho)$ in $P(S) \times P(T)$. Suppose $a \neq \lambda$. Choose $s_{1}$, $s_{2}$ in $S$ with $\alpha\left(s_{1}\right)>\lambda\left(s_{1}\right)$ and $\alpha\left(s_{2}\right)<\lambda\left(s_{2}\right)$. Set

$$
\epsilon=\min \left\{\alpha\left(s_{1}\right)-\lambda\left(s_{1}\right), \lambda\left(s_{2}\right)-\alpha\left(s_{2}\right)\right\} .
$$

Now define $a^{\prime}$ by $\alpha^{\prime}(s)=\alpha(s)$ if $s \neq s_{1}$ and $s \neq s_{2}$ and $\alpha^{\prime}\left(s_{1}\right)=\alpha\left(s_{1}\right)+$ $\epsilon$ and $\alpha^{\prime}\left(s_{2}\right)=\alpha\left(s_{2}\right)-\epsilon$. Then $\alpha$ and $\alpha^{\prime}$ differ at exactly two points and $\|a-\lambda\|=\left\|\alpha-a^{\prime}\right\|+\left\|\alpha^{\prime}-\lambda\right\|$. By repeatedly applying the above, we obtain $a_{0}, \ldots, a_{n}$ in $P(S)$ such that $a_{0}=a, a_{n}=\lambda,\|\alpha-\lambda\|=\Sigma\left\|\alpha_{i-1}-a_{i}\right\|$ and such that $\alpha_{i-1}$ and $\alpha_{i}$ differ at exactly two points.

If we choose $\nu_{1}, \ldots, \nu_{n}$ in $P(S \times T)$ such that $\pi \nu_{i}=\left(\alpha_{i}, \beta\right)$ and $\left\|\nu_{i-1}-\nu_{i}\right\|=\left\|\alpha_{i-1}-\alpha_{i}\right\|$ where $\nu_{0}=\mu$, then $\nu_{n}$ satisfies $\pi \nu_{n}=(\lambda, \beta)$ and $\left\|\mu-\nu_{n}\right\| \leq\|\alpha-\lambda\|$. Now apply the above process to $\rho$ and $\beta$ to obtain $\nu \epsilon$ 
$P(S \times T)$ such that $\pi \nu=(\lambda, \rho)$ and $\left\|\nu_{n}-\nu\right\| \leq\|\beta-\rho\|$. Then $\|\mu-\nu\| \leq$ $\left\|\mu-\nu_{n}\right\|+\left\|\nu_{n}-\nu\right\| \leq\|\alpha-\lambda\|+\|\beta-\rho\|$.

The above combinatorial result extends to the case where $S$ and $T$ are Hausdorff spaces, since the set of finite convex combinations of point mass measures on $S$ is weak ${ }^{*}$ dense in $P(S)$.

Theorem 2.2. Let $\mu \in P(S \times T)$ and set $\pi(\mu)=(\alpha, \beta)$. If $(\lambda, \rho) \in P(S)$ $\times P(T)$, then there exists $\nu \in P(S \times T)$ satisfying $\pi(\nu)=(\lambda, \rho)$ and $\|\mu-\nu\|$ $\leq\|\alpha-\lambda\|+\|\beta-\rho\|$. Hence, $\pi$ is a norm open mapping of $P(S \times T)$ onto $P(S) \times P(T)$.

Proof. Let $D(S)$ denote the family of finite decompositions $\left\{U_{1}, \ldots\right.$, $\left.U_{n}\right\}$ of $S$ where $U_{1}, \ldots, U_{n}$ are nonempty, disjoint Borel subsets of $S$. Given decompositions $\left\{A_{1}, \ldots, A_{n}\right\}$ and $\left\{B_{1}, \ldots, B_{m}\right\}$ of $S$, we write $\left\{A_{1}, \ldots, A_{n}\right\} \leq\left\{B_{1}, \ldots, B_{m}\right\}$ if each $B_{j}$ is contained in some $A_{i}$. The relation $\leq$ directs $\mathscr{D}(S)$. Given $\mathcal{U}=\left\{U_{1}, \ldots, U_{n}\right\}$ in $\mathscr{D}(S)$ and $\mathcal{O}^{2}=\left\{V_{1}, \ldots\right.$ $\left.V_{m}\right\}$ in $\mathcal{D}(T)$, choose $x_{i} \in U_{i}$ and $y_{i} \in V_{j}$. Fix $\mu \in P(S \times T)$ and $(\lambda, \rho) \epsilon$ $P(S) \times P(T)$ and set $\pi(\mu)=(\alpha, \beta)$. Given $U=\left\{U_{1}, \ldots, U_{n}\right\}$ in $\mathscr{D}(S)$ and $\mathcal{C}=\left\{v_{1}, \ldots, v_{m}\right\}$ in $\mathscr{D}(T)$ with choice points $x_{1}, \ldots, x_{n}$ and $y_{1} \ldots, y_{m}$, define

$$
\mu(\mathcal{U}, \mathcal{C})=\sum_{i, j} \mu\left(U_{i} \times V_{j}\right) \cdot \delta\left(x_{i}, y_{j}\right)
$$

where $\delta\left(x_{i}, y_{i}\right)$ is the point mass at $\left(x_{i}, x_{j}\right)$. Thus, $\left(\mathcal{U}, \mathcal{C}^{\mathbb{O}} \rightarrow \mu\left(\mathcal{U}, \mathcal{C}^{\mathbb{C}}\right)\right.$ is a net and $\mu(\mathcal{U}, \mathcal{O})$ converges to $\mu$ weak*. Likewise, define $\alpha(\mathcal{U})=$ $\Sigma \alpha\left(U_{i}\right) \delta\left(x_{i}\right)$ and $\beta\left(\mathcal{C}^{\natural}\right)=\Sigma \beta\left(V_{j}\right) \delta\left(y_{j}\right)$ and similarly for $\lambda(\mathcal{U})$ and $\rho(\mathcal{C})$.

We obtain $\nu$ as follows. By Lemma 5.1, there exists $\nu_{\mathcal{U}, 0}$ such that $\pi\left(\nu_{\mathrm{u}, \mathcal{O}}\right)=\left(\lambda(\mathcal{U}), \rho\left(\mathcal{C}^{0}\right)\right)$ and

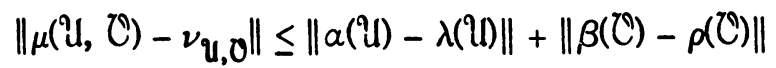

$$
\begin{aligned}
& \leq\|\alpha-\lambda\|+\|\beta-\rho\| \text {. }
\end{aligned}
$$

The net $\nu_{\mathcal{q}, \mathcal{O}}$ has a limit point $\nu$ since $P(S \times T)$ is weak ${ }^{*}$ compact. Necessarily, we have $\nu_{u, 0}$ converges to $\nu$ weak ${ }^{*}$. Hence, by weak ${ }^{*}$ continuity of $\pi$, we have $\pi(\nu)=(\lambda, \rho)$. Also, $\|\mu-\nu\| \leq\|\alpha-\lambda\|+\|\beta-\rho\|$.

We next prove that $\pi$ is weak ${ }^{*}$ open. This requires a more delicate argument but again the proof rests upon Lemma 2.1 .

Theorem 2.3. Let $S$ and $T$ be compact Hausdorff spaces. Equip $P(S)$, $P(T)$ and $P(S \times T)$ with the weak ${ }^{*}$ topology. The mapping $\pi: P(S \times T) \rightarrow$ $P(S) \times P(T)$ is weak* open. 
Proof. Fix $\mu \in P(S \times T)$ and set $\pi(\mu)=(\alpha, \beta)$. Let $F_{k} \in C(S \times T)$ such that $0 \leq F_{k} \leq 1$ for $k=1, \ldots, n$. Fix $1>\epsilon>0$. Set $\Omega=\{\nu \in P(S \times T)>$ $\left|(\mu-\nu) F_{k}\right|<18 \epsilon$ for $\left.k=1, \ldots, n\right\}$. It suffices to show that $\pi(\Omega)$ is a weak* neighborhood of $(\alpha, \beta)$. There exist closed disjoint subsets $K_{1}, \ldots, K_{p}$ of $S$ and $L_{1}, \ldots, L_{q}$ of $T$ satisfying (1) $\omega\left(F_{k}, K_{i} \times L_{j}\right)<\epsilon / 2$ where $\omega\left(F_{k}, K_{i} \times L_{j}\right)$ is the oscillation of $F_{k}$ on $K_{i} \times L_{j}$ and (2) $\mu(K \times L)>1-$ $\epsilon / 2$ where $K=U K_{i}$ and $L=U L_{j}$. Now choose $G_{k}$ continuous on $S \times T$ such that $0 \leq G_{k} \leq 1, G_{k}$ is constant on a neighborhood of $K_{i} \times L_{j}$ for each $i$ and $j$ and $\left\|F_{k}-G_{k}\right\|<\epsilon / 2$. Thus, $\left\{\nu:\left|(\mu-\nu) G_{k}\right|<17 \epsilon\right.$ for each $k=$ $1, \ldots, n\}$ is contained in $\Omega$. Choose disjoint open sets $U_{1} \ldots, U_{p}$ and $V_{1}, \ldots, V_{q}$ such that $K_{i} \subseteq U_{i}, L_{j} \subseteq V_{j}$ and $G_{k}$ is constant on $U_{i} \times V_{j}$ for each $i, j$ and $k$. Set $U=U U_{i}$ and $V=U V_{j}$. Choose functions $f_{1}, \ldots, f_{p}$ in $C(S)$ and $g_{1}, \ldots, g_{q}$ in $C(T)$ satisfying $0 \leq f_{i} \leq 1, f_{i}=1$ on $K_{i}$ and $f_{i}=0$ off $U_{i}$ for $i=1, \ldots, p$ and similarly for $g_{1}, \ldots, g_{q}$.

Now assume $(\lambda, \rho) \in P(S) \times P(T)$ and satisfies $\Sigma_{i}\left|(\alpha-\lambda) f_{i}\right|<\epsilon / 2$ and $\Sigma_{j}\left|(\beta-\rho) g_{j}\right|<\epsilon / 2$. The proof will be completed if we produce $\nu$ such that $\pi(\nu)=(\lambda, \rho)$ and $\left|(\mu-\nu) G_{k}\right|<17 \epsilon$ for $k=1, \ldots, n$. We approximate $\mu, \rho, \lambda$ in norm and apply the above lemma. First, notice that $\alpha(K)-\lambda(U) \leq$ $(\alpha-\lambda)\left(\Sigma f_{i}\right)<\epsilon / 2$. Thus, $1<\alpha(K)+\epsilon / 2<\lambda(U)+\epsilon$ and so $\lambda(U)>1-\epsilon$. Similarly, $\rho(V)>1-\epsilon$. Now define $\bar{\lambda}$ and $\bar{\rho}$ by $\lambda(U) \cdot \bar{\lambda}=\lambda \mid U$ and $\rho(V) \cdot \bar{\rho}$ $=\rho \mid V$. Define $\bar{\mu}$ by $\mu(K \times L) \cdot \bar{\mu}=\mu \mid(K \times L)$. If $\theta$ is a probability measure and $E$ is a $\theta$-measurable set with $\theta(E)>0$, then $\theta(E) \cdot \bar{\theta}=\theta \mid E$ implies $\|\theta-\bar{\theta}\|=2[1-\theta(E)]$. Hence, $\|\mu-\bar{\mu}\|<\epsilon,\|\lambda-\bar{\lambda}\|<2 \epsilon$ and $\|\rho-\bar{\rho}\|<2 \epsilon$.

It suffices to find $\bar{\nu}$ satisfying $\left|(\bar{\mu}-\bar{\nu}) G_{k}\right|<14 \epsilon$ for $k=1, \ldots, n$ and $\pi(\bar{\nu})$ $=(\bar{\lambda}, \bar{\rho})$. To see that this is sufficient, set $m=\min \{\lambda(U), \rho(V)\}$. Then $\|\lambda-m \bar{\lambda}\|=1-m<\epsilon$. Similarly, $\|\rho-m \bar{\rho}\|<\epsilon$. Finally, set

$$
\nu=m \bar{\nu}+(1-m)^{-1} \cdot(\lambda-m \bar{\lambda}) \times(\rho-m \bar{\rho}) .
$$

We have $\pi(\nu)=(\lambda, \rho)$. Also,

$$
\left|(\mu-\nu) G_{k}\right| \leq\|\mu-\bar{\mu}\|+\left|(\bar{\mu}-\bar{\nu}) G_{k}\right|+\|\nu-\bar{\nu}\|<17 \epsilon .
$$

We now construct $\bar{\nu}$. Set $\pi(\bar{\mu})=(\bar{\alpha}, \bar{\beta})$. Then $\|\alpha-\bar{\alpha}\|<\epsilon$ and $\|\beta-\bar{\beta}\|$

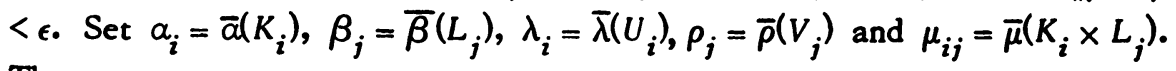
Then

$$
\begin{aligned}
\alpha_{i}-\lambda_{i} & =\bar{\alpha}\left(K_{i}\right)-\bar{\lambda}\left(U_{i}\right) \\
& =(\bar{\alpha}-\alpha) f_{i}+(\alpha-\lambda) f_{i}+(\lambda-\bar{\lambda}) f_{i}+\bar{\lambda}\left(f_{i}\right)-\bar{\lambda}\left(U_{i}\right)
\end{aligned}
$$

Summing from $i=1, \ldots, p$ and using $\Sigma \bar{\lambda}\left(U_{i}\right)=1>\bar{\lambda}\left(\Sigma f_{i}\right)$, we obtain 


$$
\begin{aligned}
\sum\left|\alpha_{i}-\lambda_{i}\right| \leq & |\bar{\alpha}-\alpha|\left(\sum f_{i}\right)+|\lambda-\bar{\lambda}|\left(\sum f_{i}\right) \\
& +\sum\left|(\alpha-\lambda) f_{i}\right|+1-\bar{\lambda}\left(\sum f_{i}\right) \\
& <\epsilon+2 \epsilon+\epsilon / 2+1-\bar{\lambda}\left(\sum f_{i}\right) .
\end{aligned}
$$

We now estimate the last term. Namely, we have

$$
\begin{aligned}
1-\bar{\lambda}\left(\sum f_{i}\right) & =(\bar{\alpha}-\alpha)\left(\sum f_{i}\right)+(\alpha-\lambda)\left(\sum f_{i}\right)+(\lambda-\bar{\lambda})\left(\sum f_{i}\right) \\
& <\epsilon+\epsilon / 2+2 \epsilon .
\end{aligned}
$$

Thus, $\Sigma\left|\alpha_{i}-\lambda_{i}\right|<7 \epsilon$. Similarly, $\Sigma\left|\beta_{j}-\rho_{j}\right|<7 \epsilon$. Apply Lemma 5.1 to obtain a doubly stochastic matrix $\left[\nu_{i j}\right]$ such that $\Sigma_{i, j}\left|\mu_{i j}-\nu_{i j}\right|<14 \epsilon, \lambda_{i}=$ $\Sigma_{j} \nu_{i j}$ and $\rho_{j}=\Sigma_{i} \nu_{i j}$. Define

$$
\bar{\nu}=\sum_{i, j} \nu_{i j}\left(\bar{\lambda} \mid U_{i}\right) \times\left(\bar{\rho} \mid V_{j}\right) / \lambda_{i} \rho_{j}
$$

Then $\pi(\bar{\nu})=(\bar{\lambda}, \bar{\rho})$. Also,

$$
\left|(\bar{\mu}-\bar{\nu}) G_{k}\right|=\left|\sum_{i, j} g_{i j}^{k}\left(\mu_{i j}-\nu_{i j}\right)\right|<14 \epsilon
$$

where $g_{i j}^{k}$ is the constant value of $G_{k}$ on $U_{i} \times V_{j^{*}}$. This completes the proof.

If one considers $\pi$ on the cone of nonnegative measures on $S \times T$, then one obtains the following open mapping results.

Theorem 2.4. Let $\mu \in M^{+}(S \times T)$ and set $\pi \mu=(\alpha, \beta)$.

(1) If $(\lambda, \rho) \in M^{+}(S, T)$, then there exists $\nu \in M^{+}(S \times T)$ satisfying $\pi \nu=(\lambda, \rho)$ and $\|\mu-\nu\| \leq 3(\|\alpha-\lambda\|+\|\beta-\rho\|) / 2$.

(2) The mapping $\pi: M^{+}(S \times T) \rightarrow M^{+}(S, T)$ is weak* open.

A proof for the first part may be obtained by establishing an appropriate variant of Lemma 2.1 [see Theorem 3.2(2) below], and taking limits in the weak* topology. The second part follows from Theorem 2.3.

3. Integrable functions on a probability space. Let $(X, \mu)$ be a probability space and let $P(X, \mu)=\left\{f \in L_{1}(X, \mu): f \geq 0\right.$ and $\left.\|f\|=1\right\}$ denote the nonnegative integrable functions on $X$ of mass 1 . We obtain open mapping results for the space $P(X, \mu)$ which are analogs of chose in $\$ 2$ and in [1]. We first consider a naturally induced operator of fundamental importance in probability theory. Let $(X, \mathcal{G}, \mu)$ be a probability space and let $B$ be a $\sigma$ subalgebra of $\mathcal{G}$. Let $\pi: L_{1}(X, \mathcal{Q}, \mu) \rightarrow L_{1}(X, \mathfrak{B}, \mu)$ be the conditional expectation operator for $\mathfrak{B}$. If $\sigma$ denotes the inclusion mapping of $L_{1}(X, \mathfrak{B}, \mu)$ into $L_{1}(X, Q, \mu)$, then $\pi$ is the continuous extension of the adjoint $\sigma^{*}$ : $L_{\infty}(X, \mathcal{Q}, \mu) \rightarrow L_{\infty}(X, \mathfrak{B}, \mu)$. See Moy [4]. 
Theorem 3.1. Let $(X, \mathcal{G}, \mu)$ be a probability space and let $\mathbb{B}$ be a $\sigma$ subalgebra of $\mathcal{A}$. Let $\pi$ denote the conditional expectation operator for $\mathfrak{B}$.

(1) $\pi$ preserves order intervals, i.e., if $f \in L_{1}^{+}(X, G, \mu)$ and if $\pi f \geq g$ $\geq 0$ where $g \in L_{1}^{+}(X, B, \mu)$, then there exists $f \geq h \geq 0$ satisfying $\pi h=g$.

(2) Fix $f \in L_{1}^{+}(X, \mathbb{Q}, \mu)$ and $g \in L_{1}^{+}(X, \mathfrak{B}, \mu)$. There exists $h \in$ $L_{1}^{+}(X, \mathcal{G}, \mu)$ satisfying $\pi h=g$ and $\|f-h\|=\|\pi f-g\|$. Hence, $\pi: L_{1}^{+}(X, \mathfrak{G}, \mu)$ $\rightarrow L_{1}^{+}(X, B, \mu)$ is norm open.

(3) $\pi: L_{1}^{+}(X, \mathbb{Q}, \mu) \rightarrow L_{1}^{+}(X, \mathfrak{B}, \mu)$ is open with respect to the weak topology.

(4) Let $S$ and $T$ denote the maximal ideal spaces of $L_{\infty}(X, Q, \mu)$ and $L_{\infty}(X, B, \mu)$, respectively. There exists $\phi: S \rightarrow T$ such that $\phi$ is continuous and open and such that $(\sigma g)^{\wedge}=\hat{g} \circ \phi$ for each $g \in L_{\infty}(X, \mathfrak{B}, \mu)$ where ^ is the Gelfand transform and $\sigma$ is the inclusion mapping of $L_{\infty}(X, Q, \mu)$.

Proof. To verify (1), fix $f \in L_{1}^{+}(x, \mathcal{G}, \mu)$ and assume $g \in L_{1}^{+}(x, \mathfrak{B}, \mu)$ where $\pi f \geq g$. Set $f_{0}=0$ and $g_{0}=0$. Now define $f_{n}$ and $g_{n}$ for $n=1,2, \ldots$ by the equations

$$
f_{n+1}=\left(f-\sum_{i=0}^{n} f_{i}\right) \wedge \sigma\left(g-\sum_{i=0}^{n} g_{i}\right) \text { and } g_{n+1}=\pi f_{n+1} \text {. }
$$

Now set $f_{\infty}=\sum_{i=1}^{\infty} f_{i}$ and $g_{\infty}=\sum_{i=1}^{\infty} g_{i}$. We have $f \geq f_{\infty}$ and $g \geq g_{\infty}$. Since $\left(f-f_{\infty}\right) \wedge \sigma\left(g-g_{\infty}\right)=0$ and $\pi\left(f-f_{\infty}\right) \geq g-g_{\infty}$, we have $g=g_{\infty}$. Thus, $\pi$ preserves order intervals. Also, notice that $(1) \Rightarrow(2)$.

Fix $f \in P(X, \mathfrak{Q}, \mu)$ and let $\mathcal{U}$ be a weak neighborhood of $f$ in $P(X, \mathfrak{Q}, \mu)$. To verify (3), we only need to show that $\pi \mathcal{U}$ is a weak neighborhood of $\pi f$ in $P(X, \mathfrak{B}, \mu)$. Choose disjoint sets $E_{1}, \ldots, E_{n}$ in $\mathbb{Q}$ which cover $X$ and $\epsilon>$ 0 satisfying $\left\{g \in P(X, \mathcal{Q}, \mu):\left|\left\langle g-f, \chi E_{i}\right\rangle\right|<\epsilon\right.$ for $\left.i=1, \ldots, n\right\} \subseteq \mathcal{U}$ and $\lambda_{i}=$ $\left.\int E_{i} f d \mu\right\rangle 0$ for $i=1, \ldots, n$. We define $\langle h, k\rangle=\int h \cdot k d \mu$ for $h \in L_{1}$ and $k \in L_{\infty}$. Set $f_{i}=\lambda_{i}^{-1} f \cdot \chi E_{i^{*}}$. Then $\Sigma_{i=1}^{n} \lambda_{i} f_{i}=f$ and $\Sigma_{i=1}^{n} \lambda_{i}=1$ and $f_{i} \epsilon$ $P(X, \mathbb{Q}, \mu)$. Now set $\mathcal{U}_{j}=\left\{g \in P(X, \mathbb{G}, \mu):\left|\left\langle g-f_{j}, \chi E_{i}\right\rangle\right|<\epsilon\right.$ for $i=1, \ldots$, $n$ \}. Then $\lambda_{1} \mathcal{U}_{1}+\cdots+\lambda_{n} \mathcal{U}_{n} \subseteq \mathcal{U}$. Since $f_{j} \cdot \chi E_{i}=0$ if $i \neq j$, we have that $\mathcal{U}_{j}=\left\{g \in P(X, Q, \mu):\left|\left\langle g, X E_{j}\right\rangle\right|>1-\epsilon\right\}$ and so, by applying (1), that $\pi U_{j}$ is a weak neighborhood of $\pi f_{j}$ in $P(X, \Re, \mu)$. It remains to show that if $\mathcal{C}_{1}^{j}$ and $C_{2}$ are weak neighborhoods of $g_{1}$ and $g_{2}$ in $P(X, B, \mu)$ and if $a_{1}+a_{2}=1$ where $a_{1}, a_{2}>0$, then $a_{1} \mathbb{C}_{1}$ and $a_{2} \mathcal{C}_{2}$ is a weak neighborhood of $\alpha_{1} g_{1}+$ $a_{2} g_{2}$. Fix $\psi_{1}, \ldots, \psi_{m} \in L_{\infty}(X, \mathfrak{B}, \mu)$ such that $\left\|\psi_{j}\right\|_{\infty} \leq 1$ and $\mathfrak{C}_{i} \supseteq$ $\left\{g \in P(X, B, \mu):\left|\left\langle g-g_{i}, \psi_{j}\right\rangle\right|<\epsilon\right.$ for $\left.j=1, \ldots, m\right\}$. Set $\phi_{i}=$ $g_{i} /\left(\alpha_{1} g_{1}+\alpha_{2} g_{2}\right)$. Set $\alpha=\max \left\{\alpha_{1} / \alpha_{2}, \alpha_{2} / \alpha_{1}\right\}$. Fix $g \in P(X, B, \mu)$ satisfying 


$$
\left|\left\langle g-\left(\alpha_{1} g_{1}+\alpha_{2} g_{2}\right), \phi_{i} \psi_{j}\right\rangle\right|<\epsilon / 2 \text { and }\left\|\phi_{i} g\right\|<1+\epsilon / 2 \alpha
$$

for $i=1,2$ and $j=1, \ldots, m$. We now have $\left\langle\phi_{i} g-g_{i}, \psi_{j}\right\rangle=\left\langle g-\left(\alpha_{1} g_{1}+\right.\right.$ $\left.\left.\alpha_{2} g_{2}\right), \phi_{i} \psi_{j}\right\rangle$ and $\alpha_{1} \phi_{1} g+\alpha_{2} \phi_{2} g=g$. Assume $\beta=\left\|\phi_{1} g\right\| \geq 1$. Then set $h_{1}=\phi_{1} g / \beta$ and $h_{2}=\phi_{2}=\phi_{2} g+\alpha_{1}\left(\phi_{1} g-h_{1}\right) / a_{2}$. Then $\alpha_{1} h_{1}+\alpha_{2} h_{2}=g$ and $\left|\left\langle h_{i}-g_{i}, \psi_{j}\right\rangle\right|<\epsilon$ since $\left\|\alpha_{1}\left(\phi_{1} g-h_{1}\right) / \alpha_{2}\right\|<\epsilon / 2$ and $\left\|\phi_{1} g_{1}-h_{1}\right\|<$ $\epsilon / 2$. The case $\left\|\phi_{1} g\right\|<1$ is similar.

To prove (4), let $S^{\#}$ and $T^{\#}$ denote the open and closed subsets of $S$ and $T$, respectively. Define $\hat{\pi}: C(S) \rightarrow C(T)$ and $\hat{\sigma}: C(T) \rightarrow C(S)$ by $\hat{\pi}(\hat{f})=$ $(\pi f)^{\wedge}$ for $f \in L_{\infty}(X, \mathcal{G}, \mu)$ and $\hat{\sigma}(\hat{g})=(\sigma g)^{\wedge}$ for $g \in L_{\infty}(X, \mathfrak{B}, \mu)$. Thus, $\hat{\sigma}$ maps idempotents in $C(T)$ to idempotents in $C(S)$. Thus, we have $\Phi: T^{\#}$ $\rightarrow S^{\#}$ defined by $\Phi(E)=K$ iff $\hat{\sigma}\left(\chi_{E}\right)=\chi_{K}$. Since $\Phi$ is a Boolean algebra monomorphism of $T$ into $S, \Phi$ determines $[2, \mathrm{p} .85]$ a continuous map $\phi$ of $S$ onto $T$ where $\phi^{-1}(E)=\Phi(E)$ for each $E \in T^{\#}$. Thus, $g \circ \phi=\hat{\sigma}(g)$ for each $g \in C(T)$. To see that $\phi$ is open, fix $K$ open and closed in $S$. Let $U=\left\{t \in T:\left(\pi \chi_{K}\right)(t)>0\right\}$. Then $U$ is open in $T$ and $U \subseteq \phi(K) \subseteq \bar{U}$ where $\bar{U}$ denotes the closure of $U$. Since $T$ is extremally disconnected, we have $\bar{U}$ is open and so $\phi(K)=\bar{U}$ is open.

We now take up the problem of establishing open mapping properties for marginals of integrable functions on $(X \times Y, \mu \times \nu)$ where $(X, \mu)$ and $(Y, \nu)$ are probability spaces. Given $F \in L_{1}(X \times Y, \mu \times \nu)$, set $\pi F=\left(f_{1} f_{2}\right)$ where $f_{1}(x)=\int F(x, y) d \nu(y)$ and $f_{2}(y)=\int F(x, y) d \mu(x)$. Although closely related to Theorem 2.2, our next result requires a different proof. A straightforward application of Lemma 2.1 shows that $\pi: P(X \times Y, \mu \times \nu) \rightarrow P(X, \mu) \times P(Y, \nu)$ is open with respect to the weak topology. Likewise, in case $S$ and $T$ are totally disconnected compact spaces, one can similarly show that $\pi P(S \times T)$ $\rightarrow P(S) \times P(T)$ is weak ${ }^{*}$ open. One should also note that part (1) implies Lemma 2.1.

Theorem 3.2. Let $(X, \mu)$ and $(Y, \nu)$ be probability spaces. Let $F \epsilon$ $L_{1}^{+}(X \times Y, \mu \times \nu)$ and set $\pi F=\left(f_{1}, f_{2}\right)$.

(1) Assume $\|F\|=1$ and $\left(g_{1}, g_{2}\right) \in P(X, \mu) \times P(Y, \nu)$. Then there exists $G \geq 0$ such that $\pi G=\left(g_{1}, g_{2}\right)$ and $\|F-G\| \leq\left\|f_{1}-g_{1}\right\|+\left\|f_{2}-g_{2}\right\|$.

(2) Fix nonnegative marginals $\left(g_{1}, g_{2}\right)$. There exists $G \geq 0$ such that $\pi G=\left(g_{1}, g_{2}\right)$ and $\|F-G\| \leq 3\left(\left\|f_{1}-g_{1}\right\|+\left\|f_{2}-g_{2}\right\|\right) / 2$.

(3) The mapping $\pi: P(X \times Y, \mu \times \nu) \rightarrow P(X, \mu) \times P(Y, \nu)$ is open with respect to the weak topology.

Proof. As noted above, the proof of (3) is straightforward. Fix $F \geq 0$ and set $\pi F=\left(f_{1}, f_{2}\right)$. Let $\left(g_{1}, g_{2}\right)$ be nonnegative marginals. We first 
consider the case where $f_{2}=g_{2}$. By Theorem 3.1(1), there exists $0 \leq H \leq F$ such that $\pi H=\left(h_{1}, h_{2}\right)$ where $h_{1}=f_{1} \wedge g_{1}$. We may certainly assume $\left\|f_{1}-h_{1}\right\|=\lambda>0$. Set $G=H+\left(g_{1}-h_{1}\right)\left(g_{2}-h_{2}\right) / \lambda$. One obtains $\pi G=$ $\left(g_{1}, g_{2}\right)$ and $\|F-G\|=2\left\|g_{1}-h_{1}\right\|=\left\|f_{1}-g_{1}\right\|$. The case $f_{1}=g_{1}$ is handled similarly. To see (1), simply consider the intermediate marginals $\left(f_{1}, g_{2}\right)$ and apply the above two cases.

We next consider the case $\left(g_{1}, g_{2}\right) \leq\left(f_{1}, f_{2}\right)$ which denotes $g_{1} \leq f_{1}$ and $g_{2} \leq f_{2}$. Choose $F \geq B \geq 0$ such that $B$ is maximal with respect to property $\pi B \leq\left(g_{1}, g_{2}\right)$. Set $\pi B=\left(b_{1}, b_{2}\right)$ and $h_{i}=g_{i}-b_{i}$. We may assume $\left\|h_{i}\right\|>0$. Next, choose $F-B \geq C \geq 0$ such that $C$ is maximal with respect to the property $\pi(F-B-C) \geq\left(b_{1}, h_{2}\right)$. If we set $A=F-B-C$ and $\pi A=\left(a_{1}, a_{2}\right)$ and $\pi C=\left(c_{1}, c_{2}\right)$, then we have $A=0$ on $\left[h_{1}(x)>0\right] \times\left[h_{2}(y)>0\right]$ and on $\left[h_{1}(x)=0\right] \times\left[h_{2}(y)=0\right]$ and we have $h_{i}=a_{i}$ on $\left[h_{i}>0\right]$. Thus,

$$
\left\|f_{1}-g_{1}\right\|+\left\|f_{2}-g_{2}\right\|=\left\|c_{1}\right\|+\left\|c_{2}\right\|+\left\|a_{1}-h_{1}\right\|+\left\|a_{2}-h_{2}\right\| .
$$

Then $\pi H=\left(h_{1}, h_{2}\right)$. It suffices to show

$$
\|A-H\| \leq \frac{3}{2}\left(\left\|a_{1}-b_{1}\right\|+\left\|a_{2}-b_{2}\right\|\right)
$$

since setting $G=B+H$ yields $\pi G=\left(g_{1}, g_{2}\right)$ and

$$
\|F-G\| \leq\|C\|+\|A-H\| \leq \frac{3}{2}\left(\left\|f_{1}-g_{1}\right\|+\left\|f_{2}-g_{2}\right\|\right) \text {. }
$$

Using $\left\|h_{1}\right\|=\left\|a_{2}-h_{2}\right\|$ and $\left\|h_{2}\right\|=\left\|a_{1}-h_{1}\right\|$, we have

$$
\|A-H\|=\|A\|+\|H\|=\left\|a_{1}-b_{1}\right\|+\left\|h_{2}\right\|=\frac{3}{2}\left(\left\|a_{1}-b_{1}\right\|+\left\|a_{2}-b_{2}\right\|\right) \text {. }
$$

We now complete the proof of (2). Set $k_{i}=f_{i} \wedge g_{i}$. Set $k_{i}=f_{i} \wedge g_{i}$ We may assume $\left\|k_{1}\right\| \leq\left\|k_{2}\right\|$. Fix $1 \geq \epsilon, \delta \geq 0$ such that $\left(k_{1}+\epsilon\left(f_{1}-k_{1}\right)\right.$, $\left.k_{2}\right)$, and $\left(k_{1}+\delta\left(g_{1}-k_{1}\right), k_{2}\right)$ are marginals. By the case considered above, there exists $K \geq 0$ satisfying $\pi K=\left(k_{1}+\epsilon\left(f_{1}-k_{1}\right), k_{2}\right)$ and

$$
\|F-K\| \leq \frac{3}{2}\left(\left\|f_{2}-k_{2}\right\|+(1-\epsilon)\left\|f_{1}-k_{1}\right\|\right) \text {. }
$$

Now apply (1) to obtain $H$ satisfying $\pi H=\left(k_{1}+\delta\left(g_{1}-k_{1}\right), k_{2}\right)$ and $\|K-H\|$ $\leq \epsilon\left\|f_{1}-k_{1}\right\|+\delta\left\|g_{1}-k_{1}\right\|$. Choose $G \geq 0$ satisfying $\pi G=\left(g_{1}, g_{2}\right)$ and $\|H-G\|=(1-\delta)\left\|g_{1}-k_{1}\right\|$. Then we have

$$
\|F-G\| \leq\|F-K\|+\|K-H\|+\|H-G\| \leq \frac{3}{2}\left(\left\|f_{1}-g_{1}\right\|+\left\|f_{2}-g_{2}\right\|\right) \text {. }
$$

Acknowledgement. The author wishes to thank the referee for suggestions on the presentation of the results. 


\section{REFERENCES}

1. S. Z. Ditor and L. Q. Eifler, Some open mapping theorems for measures, Trans. Amer. Math. Soc. 164 (1972), 287-293.

2. P. R。 Halmos, Lectures on Boolean algebras, Van Nostrand Math. Studies, no. 1, Van Nostrand, Princeton, N. J., 1963. MR 29 \#4713.

3. H. G. Kellerer, Masstheoretische Marginalprobleme, Math. Ann. 153 (1964), 168-198. MR 28 \#160.

4. S.-T. C. Moy, Characterizations of conditional expectation as a transformation on function spaces, Pacific J. Math. 4 (1954), 47-63. MR 15, 722.

5. V. Strassen, The existence of probability measures with given marginals, Ann. Math. Statist. 36 (1965), 423-439. MR 31 \#1693.

6. L. Q. Eifler, Open mapping theorems for probability measures on metric spaces (to be submitted to Ann. Inst. Fourier, Grenoble).

DEP ARTMENT OF MATHEMATICS, UNIVERSITY OF MISSOURI, KANSAS CITY, MISSOURI 64110 